Research Article

\title{
A New Spray Approach to Produce Uniform Ultrafine Coatings
}

\author{
Lijuan Qian $\mathbb{D}$, Shaobo Song $\mathbb{D}$, and Xiaolu Li
}

China Jiliang University, Hangzhou, China

Correspondence should be addressed to Lijuan Qian; mecqlj@126.com

Received 4 February 2018; Accepted 2 April 2018; Published 3 July 2018

Academic Editor: Xiaoke Ku

Copyright (C) 2018 Lijuan Qian et al. This is an open access article distributed under the Creative Commons Attribution License, which permits unrestricted use, distribution, and reproduction in any medium, provided the original work is properly cited.

\begin{abstract}
A new spray approach is proposed to overcome the disadvantages of the traditional single-orifice nozzle, such as uneven coatings, overspray, and low efficiency. Both the experimental measurements and numerical simulation are used to investigate the spray characteristics of the multiorifice nozzle. The results show the new nozzle structure is able to disperse the particles in a wider regime and reduce the central pressure. It is an effective way to produce uniform ultrafine coatings.
\end{abstract}

\section{Introduction}

Nanoparticles with size between a few nanometers and $100 \mathrm{~nm}$ have great potential for use in various industry applications such as nanostructured film deposition [1], drug carriers [2], superconductors [3], and catalyst. There are a large number of techniques for the preparations of ultrafine particles [4]. Among them, spray technology using suspension or solution precursors has been most widely used [5]. The suspension or solution feeding is prepared by the coating material dispersing or dissolving in a liquid carrier, which allows the controlled injection of much finer particle than conventional thermal spraying $[6,7]$.

One of the largest challenges of spray technology is how to control the spray pattern and droplet size distribution. As concern to the traditional spray technology, such as round jet and annular spray, there are some significant defects. For example, firstly, the droplets tend to concentrate in the center axis which gives rise to the highly uneven distribution of liquid fragments. Second, the pressure is too high in the spray center, which always results in overspray. Third, the spray efficiency is low since the spatial distribution is narrow. These problems are particularly important for ultrafine coating due to its strict requirements. In order to solve these problems, the common practices are optimization design of the operating parameters such as adjustment of pressure, nozzle orifice, and gas-to-liquid flow ratio [8-11]. These approaches can alleviate the uneven spray pattern, whereas the effect is limited and not essential to solve the above problems.

In this paper, a new spray approach with nozzle structure of multiple gas orifices is introduced to greatly improve the uniformity of the ultrafine coatings. Both the diagnostic measurements and numerical modeling are used to investigate the differences in flow behavior of multiorifice spray and traditional single-hole spray. For the experimental setup, hot-wire anemometer is used to measure the distributions of gas velocity, and laser particle analyzer is introduced to obtain the data of the particle size distribution. For the numerical calculation, based on the commercial software, discrete particle model (DPM) is adopted to simulate the gas-liquid two-phase flow. Taylor analogy breakup (TAB) model is used to describe the droplet breakup. The calculation results are compared by the experimental data. After validation, spatial distribution of pressure and velocity field is analyzed in detail to reveal the spray pattern characteristics of the multiorifice nozzle. Then, the particle size and spatial distributions are discussed, and the advantages of the new spray approaches are concluded.

\section{Numerical Setup}

2.1. Multiorifice Nozzle Structure. Figure 1 illustrates the structure of multiorifice nozzle. There are three types of 


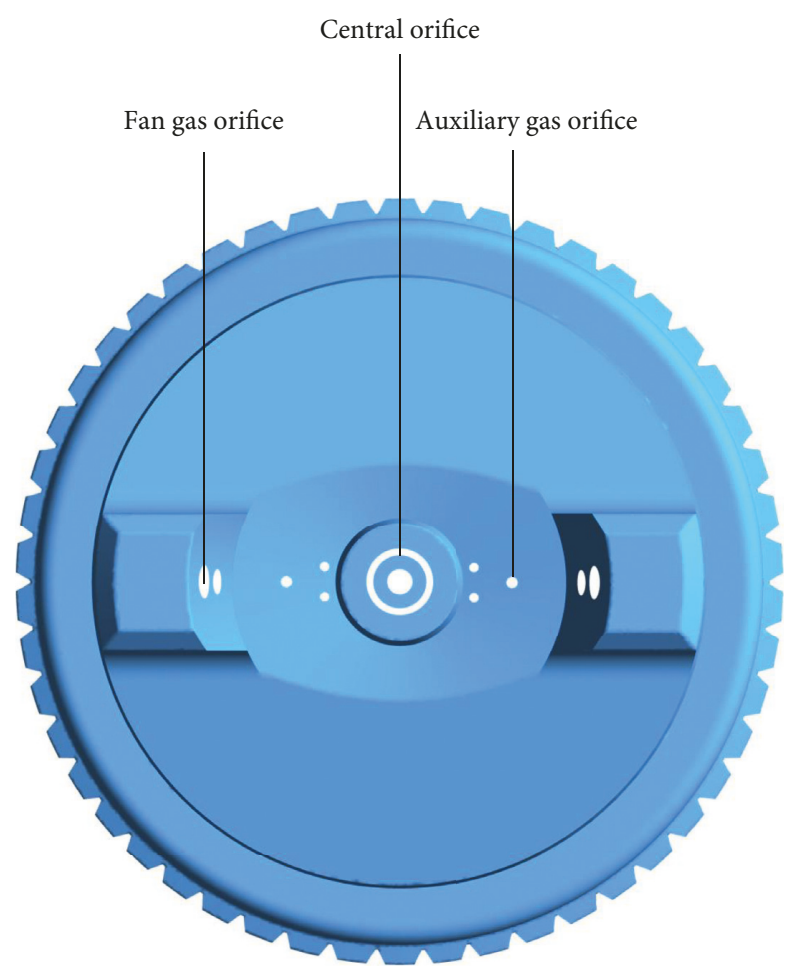

FIGURE 1: Multiorifice nozzle structure.

orifices, which include central orifice, auxiliary gas orifices, and fan gas orifices. Based on an extremely large relative velocity between gas and liquid (more than $100 \mathrm{~m} / \mathrm{s}$ ), the central orifice can be able to facilitate primary atomization. The auxiliary gas orifices are used to carry out secondary atomization and alleviate the phenomenon of entrainment. For the fan gas orifices, gas was provided at a constant slope angle. By controlling gas flux and pressures, the gas flow from fan orifice can be used to adjust the spray shape.

2.2. Computing Model. Using the commercial software, the discrete particle model (DPM) was adopted to describe the gas-liquid two-phase flow. DPM is a multiphase flow model based on the Eulerian-Lagrangian method, in which the gas governing equation is solved as a continuous phase in the Euler coordinate system $[12,13]$. The particles are considered as a discrete phase in the Lagrangian coordinate system. Initially, the gas flow field was numerically calculated by $k-\varepsilon$ turbulent model and pressure-based solver. Then, the droplet particle was added subsequently by using solid-cone injection type, dynamic drag model, and Taylor analogy breakup (TAB) model $[14,15]$. The equation of motion of particles is shown below:

$$
\frac{d \mathbf{u}_{\mathrm{p}}}{d t}=\frac{\mathbf{u}_{\mathrm{g}}-\mathbf{u}_{\mathrm{p}}}{\tau_{\mathrm{r}}}+\frac{\mathbf{g}\left(\rho_{\mathrm{p}}-\rho_{\mathrm{g}}\right)}{\rho_{\mathrm{p}}}+\Delta,
$$

where $\rho_{\mathrm{p}}$ is the density of the particle, $\rho_{\mathrm{g}}$ is the density of the fluid, $\mathbf{u}_{\mathrm{p}}=\left(u_{\mathrm{p}}, v_{\mathrm{p}}, w_{\mathrm{p}}\right)$ and $\mathbf{u}_{\mathrm{g}}=\left(u_{\mathrm{g}}, v_{\mathrm{g}}, w_{\mathrm{g}}\right)$ are, respectively, the velocity vectors of discrete phase particle and continuous phase fluid, $\mathbf{g}$ is the gravitational acceleration, and $\Delta$ is an additional acceleration term for unit particle mass. The dynamic

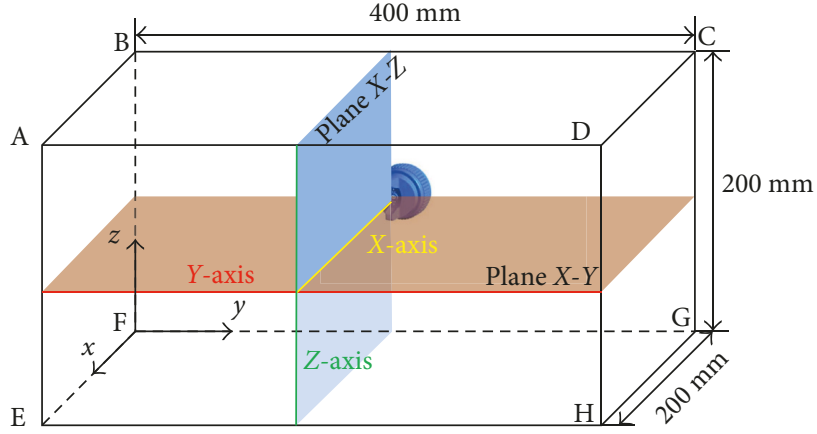

FIgURE 2: Schematic of computational domain.

drag coefficient model which is incorporated in FLUENT software has been used in $\Delta . \tau_{\mathrm{r}}$ is the discrete phase particle relaxation time. The droplet distortion equation of TAB model for droplet breakup can be expressed as below:

$$
F-k x-d \frac{d x}{d t}=m \frac{d^{2} x}{d t^{2}}
$$

where $x$ is the displacement of the droplet equator from its spherical position. The coefficients of (2) are determined by Taylor analogy [16]: $F / m=C_{\mathrm{F}} \rho_{\mathrm{g}} u^{2} / \rho_{\mathrm{p}} r, k / m=C_{\mathrm{k}} \sigma / \rho_{\mathrm{p}} r^{3}$, and $d / m=C_{\mathrm{d}} \mu_{\mathrm{p}} / \rho_{\mathrm{p}} r^{2}$.

Figure 2 shows the computational regime of the numerical model. The nozzle is located in a cuboid computational domain with size of $200 \mathrm{~mm} * 200 \mathrm{~mm} * 400 \mathrm{~mm} .21$ million computational cells were used in the whole domain. Finer meshes are used for the core region of the spray. The grid dependency has been validated, and the computational results are convergence under the present mesh size. Pressure inlet boundary conditions are applied on the orifices of the nozzle. The injection pressures of central and auxiliary gas orifices are $120 \mathrm{KPa}$ and $50 \mathrm{KPa}$ for the fan gas orifices. The wall boundary condition is used at the plane ADHE. Pressure outlet boundary conditions are applied on the planes ABCD, EFGH, DCGH, ABFE, and BCGF. For discrete particles, the parcels are introduced in the system at the central orifice exit, which has the original size of $65 \mu \mathrm{m}$ with Rosin-Rammler distribution and original velocity of $50 \mathrm{~m} / \mathrm{s}$. Usually, a total number of 10,000 computational parcels are injected into the flow for each case. These initial conditions are provided and validated by the experimental data.

\section{Experimental Setup}

3.1. Velocity Measurements. The schematic of hot-wire anemometry measuring system is shown in Figure 3. This system can be used to obtain the velocity data of gas flow filed. The gas source is provided by an air pump which can satisfy actual working conditions. The airflow pressure can be measured by the piezometer. Before measurement, the velocity calibration should be conducted. Then, a traverse measurement system is applied to acquire velocity data by a hot-wire probe. The measuring results include the velocity data on planes $X-Y$ and $X-Z$ (shown in Figure 2). 


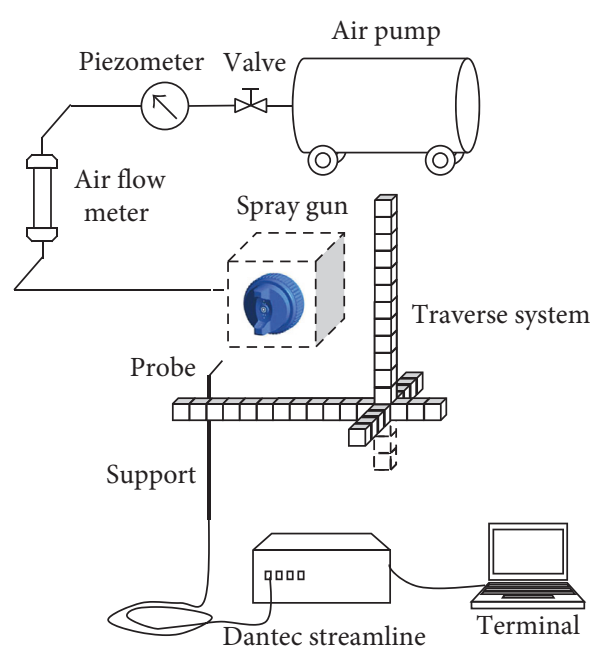

(a)

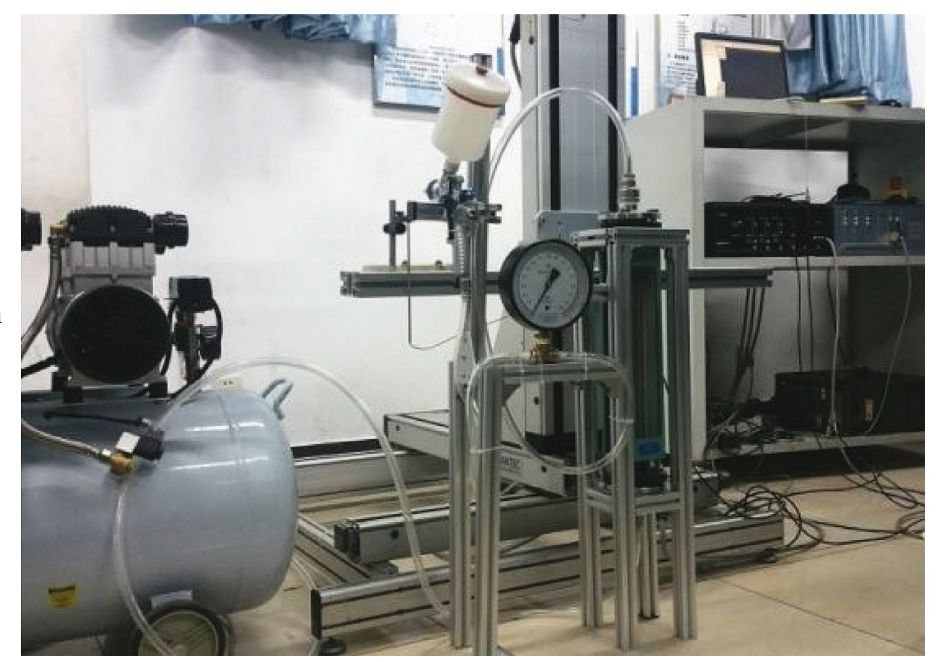

(b)

FIGURE 3: Schematic of hot-wire anemometry measuring system.

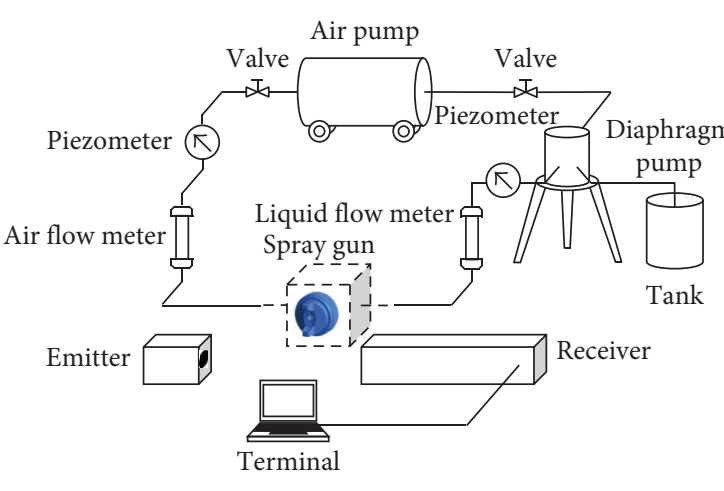

(a)

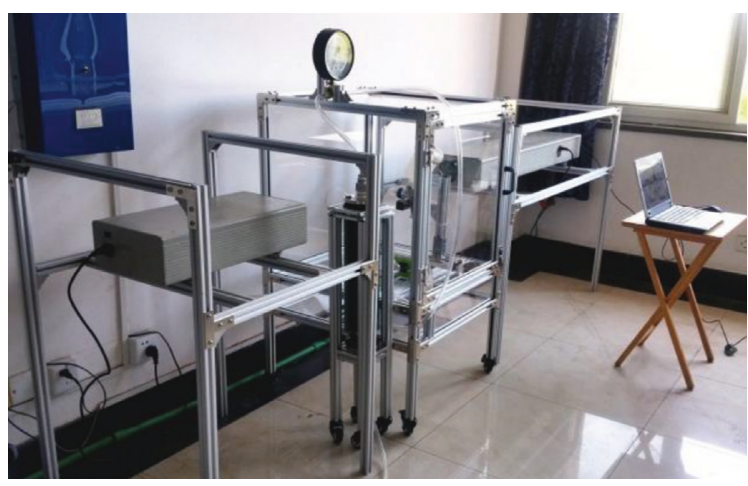

(b)

FIGURE 4: Schematic of laser particle analyzer measuring system.

3.2. Particle Size Measurements. Figure 4 illustrates the laser particle analyzer measurement system. The laser particle analyzer device includes the emitter and receiver part. These two parts are set upon the different position of spray gun to ensure laser traversing measuring area. The receiver device must be calibrated before the first measurement. The lid of the receiver is used to ensure that no natural light distorts the accuracy of measurements. A traverse coordinate system is used to measure particle size data from various cross sections. Sources of gas and liquid are provided with air pump and diaphragm pump, respectively. The pressure and flow rate can be measured by the piezometer and flow meter, respectively. The gas media is air, and the liquid media is water.

\section{Result and Discussion}

\subsection{Single-Phase Flow Field}

4.1.1. Validation. Figure 5 shows a comparison of experimental data and model predictions for the gas velocity distributions of the multiorifice nozzle. Both the experiments and calculations are conducted under the same operating conditions that are presented in Section 2.2. The agreement between the predictions and measurements is qualitatively achieved. Results show the gas velocity reaches highest in the center of the spray core at the nozzle exit. With the evolution of the spray, gas velocity declined dramatically from $70 \mathrm{~m} / \mathrm{s}$ to lower than $10 \mathrm{~m} / \mathrm{s}$. Due to the effects of fan gas orifice, the spray angle on the $X-Y$ plane is much larger than that on the $X-Z$ plane, which is totally different from the single round or annular orifice jet.

Figure 6 illustrates the quantitative comparison of the velocity evolution along with the $Y$-axis and $X$-axis. In Figure 6(a), $d$ represents the distance between the cross section and the nozzle orifice. As the $d$ increases, the velocity curve of the gas filed along with the $Y$-axis tends to be flat progressively, which means the velocity field approaches to homogenization. As Figure 6(b) indicates, the gas velocity decays at the downstream of the spray due to the entrainment effects. In Figure 6, the calculated results agreed with experimental data well. 

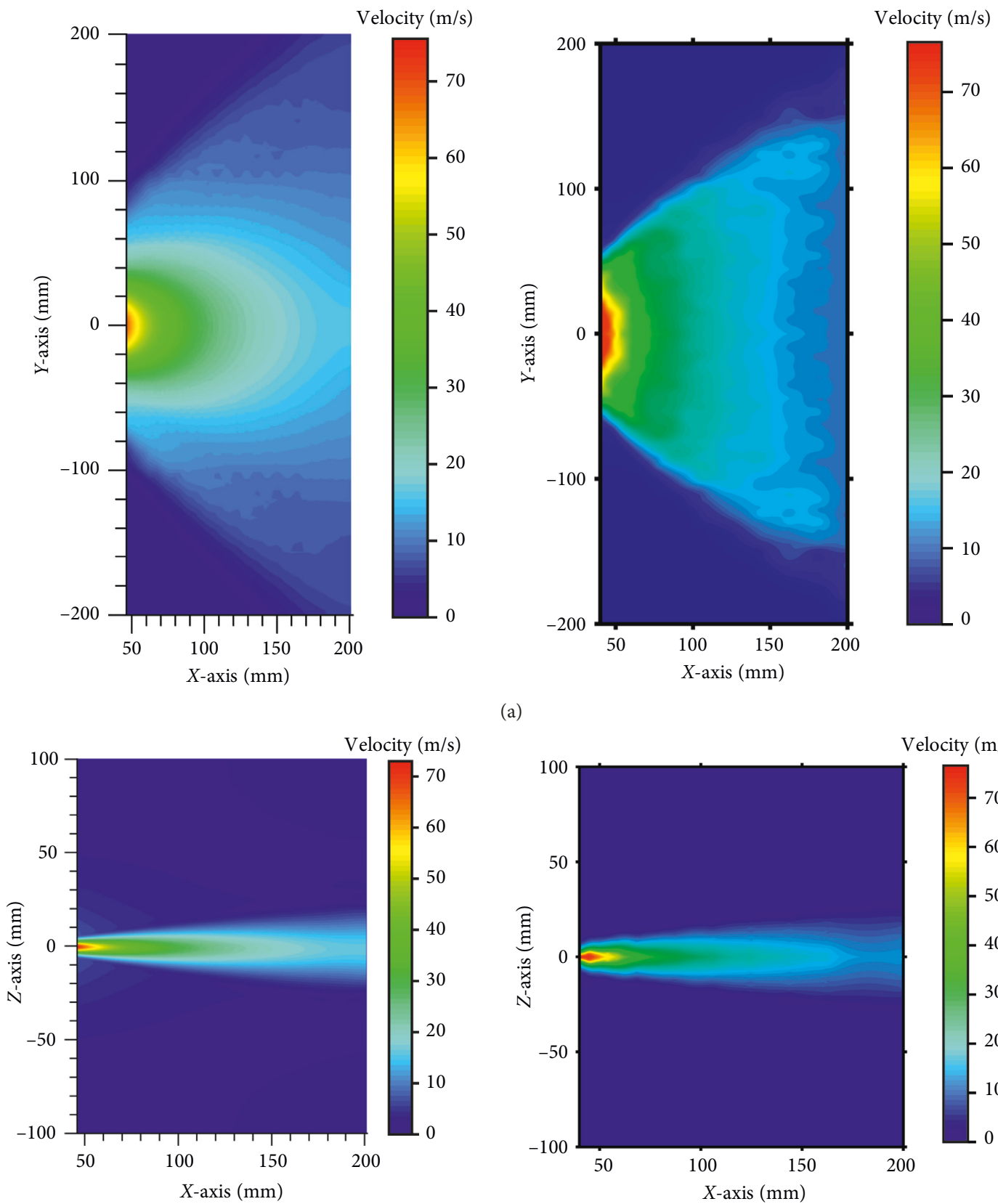

(a)

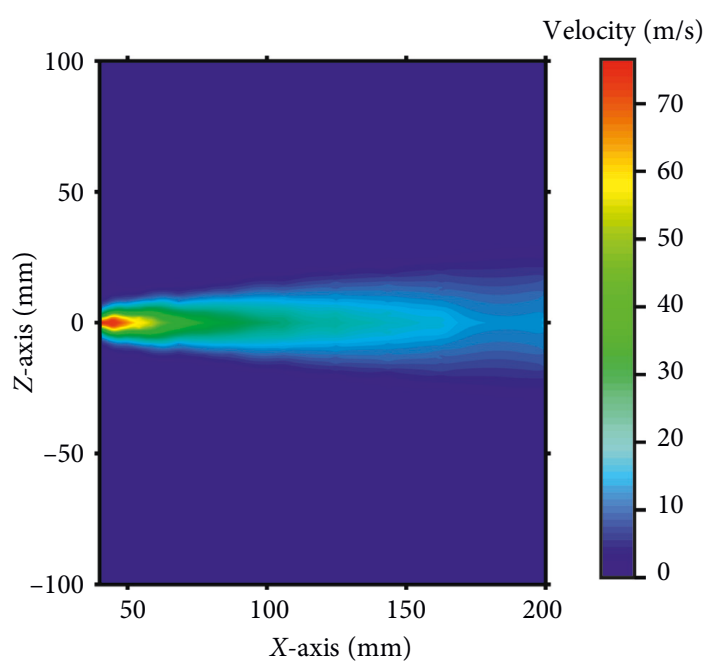

(b)

Figure 5: Comparison of computational and experimental gas velocity distribution. (a) Contours on the $X-Y$ plane (left: calculation; right: experiment); (b) contours on the $X-Z$ plane (left: calculation; right: experiment).

4.1.2. Spatial Distribution of Pressure and Velocity Field. Among the investigations of the spray filed, the distribution of pressure and velocity is the primary interest. Figures 7 and 8 compare the velocity and pressure contours between single-hole nozzle spray and multiorifice nozzle spray, respectively. In Figures $7(\mathrm{a})$ and 8(a), for the single-orifice case, the velocity and pressure contours are almost symmetrical at the $X-Y$ plane and $X-Z$ plane, whereas, for the multiorifice case, with the help of auxiliary gas orifices and fan gas orifices, the spray pattern becomes totally different. The differences can be concluded in three points: firstly, in the single-nozzle case, the jet pattern is similar to a cylinder. The spray angles for both $X-Y$ and $X-Z$ planes are small. While for the multiorifice case, the spray range becomes larger on the $X-Y$ plane after leaving the orifice for a distance. On the contrary, the spray angle becomes smaller on the $X-Z$ plane beyond $15 \mathrm{~mm}$ from the nozzle exit. Second, at the downstream of the spray, the central pressure of the multiorifice jet is lower than that of the single-orifice jet, which can relieve the phenomenon of overspray in the center. Finally, due to the effect of spray entrainment, the nozzle exits are used to be polluted by the whirling gas and 


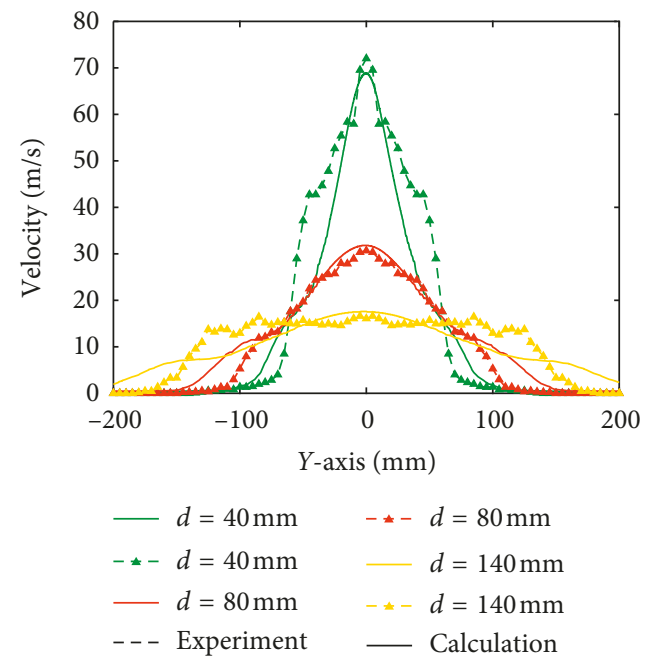

(a)

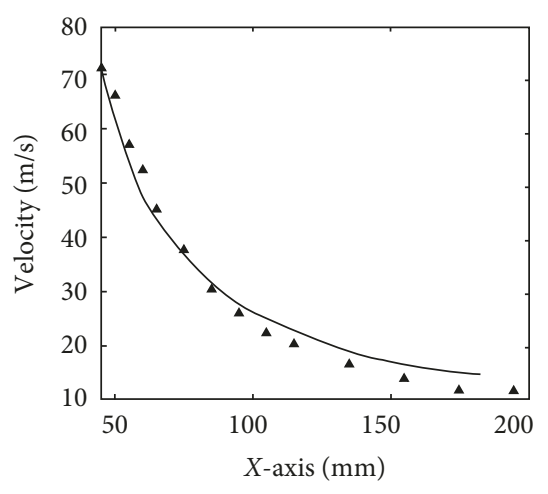

- Calculation

(b)

Figure 6: Comparison of computational and experimental gas velocity evolution. (a) Gas velocity along with the $Y$-axis for different measuring points; (b) gas velocity along with the $X$-axis.
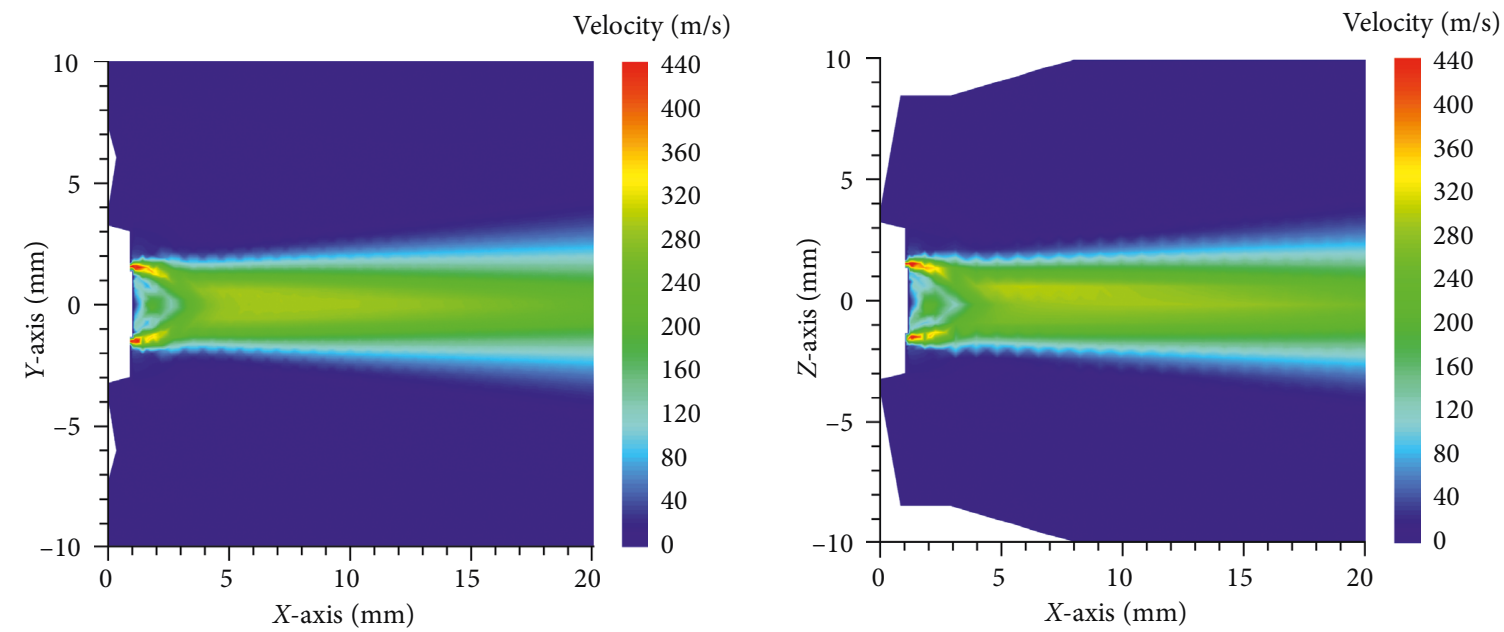

(a)
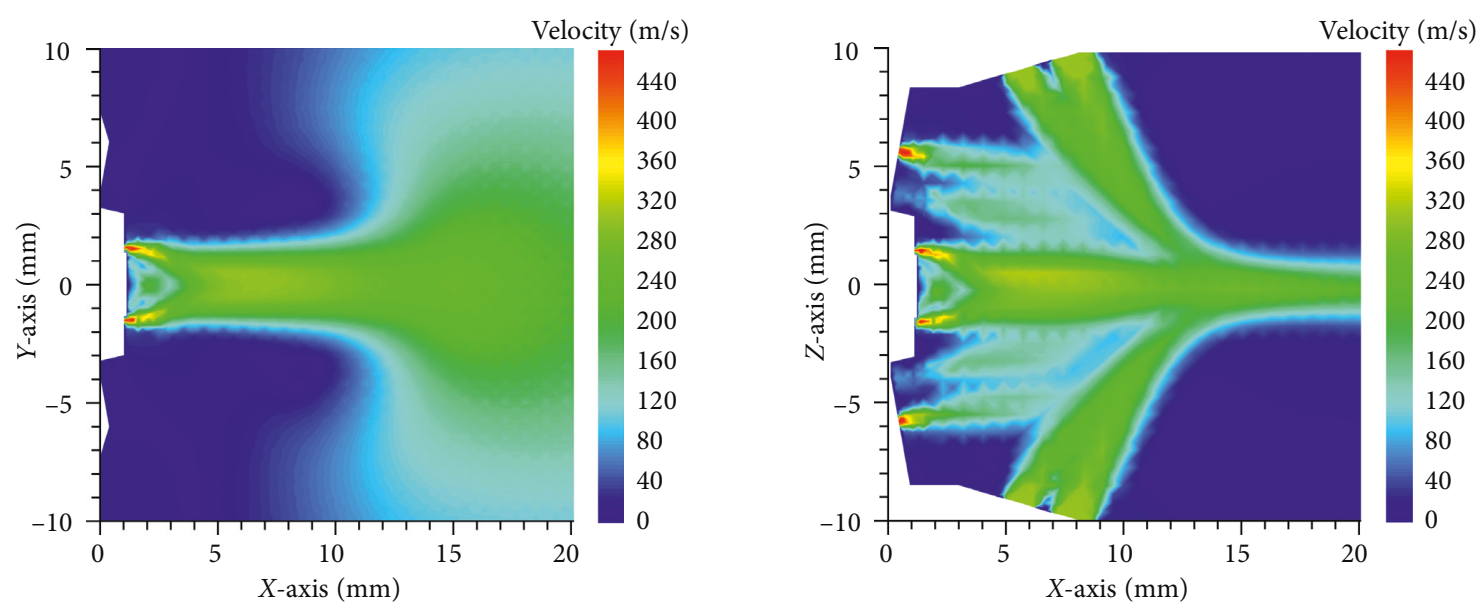

(b)

Figure 7: Comparison of calculated spatial distribution of velocity field. (a) Velocity contours for single-hole nozzle: $X$ - $Y$ plane (left); $X-Z$ plane (right). (b) Velocity contours for multiorifice nozzle: $X-Y$ plane (left); $X-Z$ plane (right). 

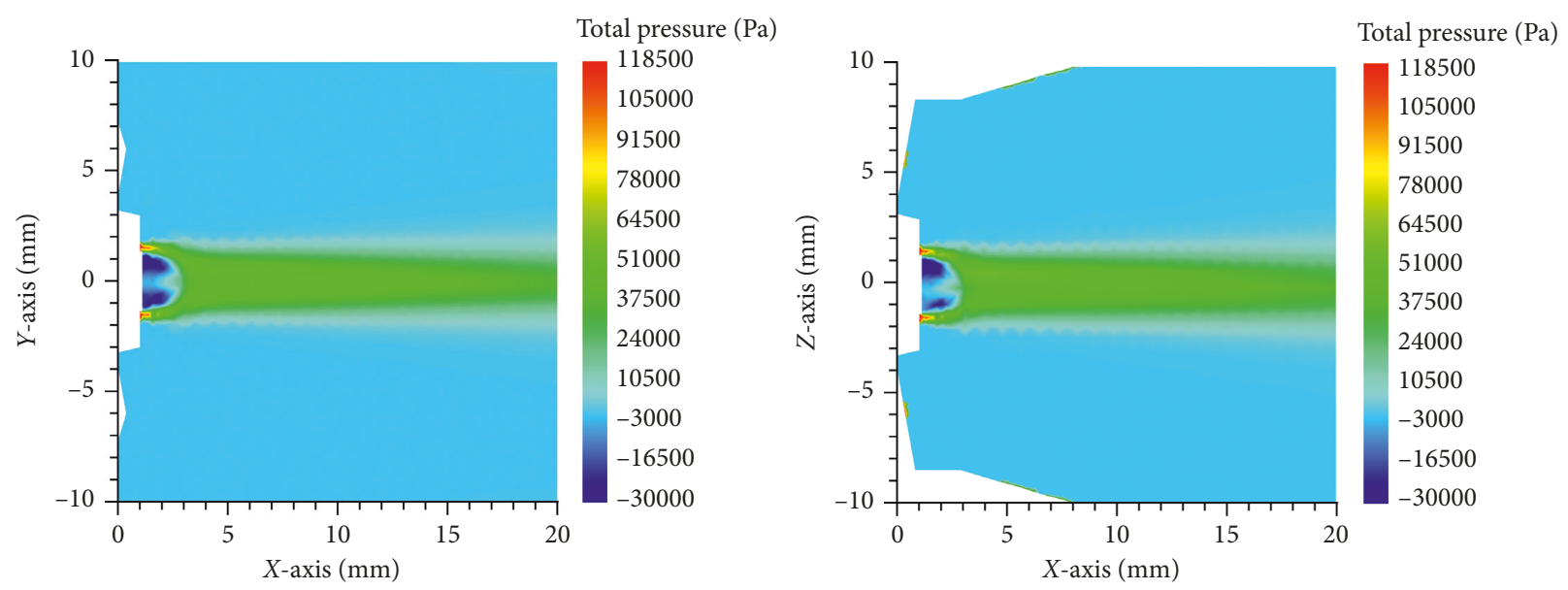

(a)
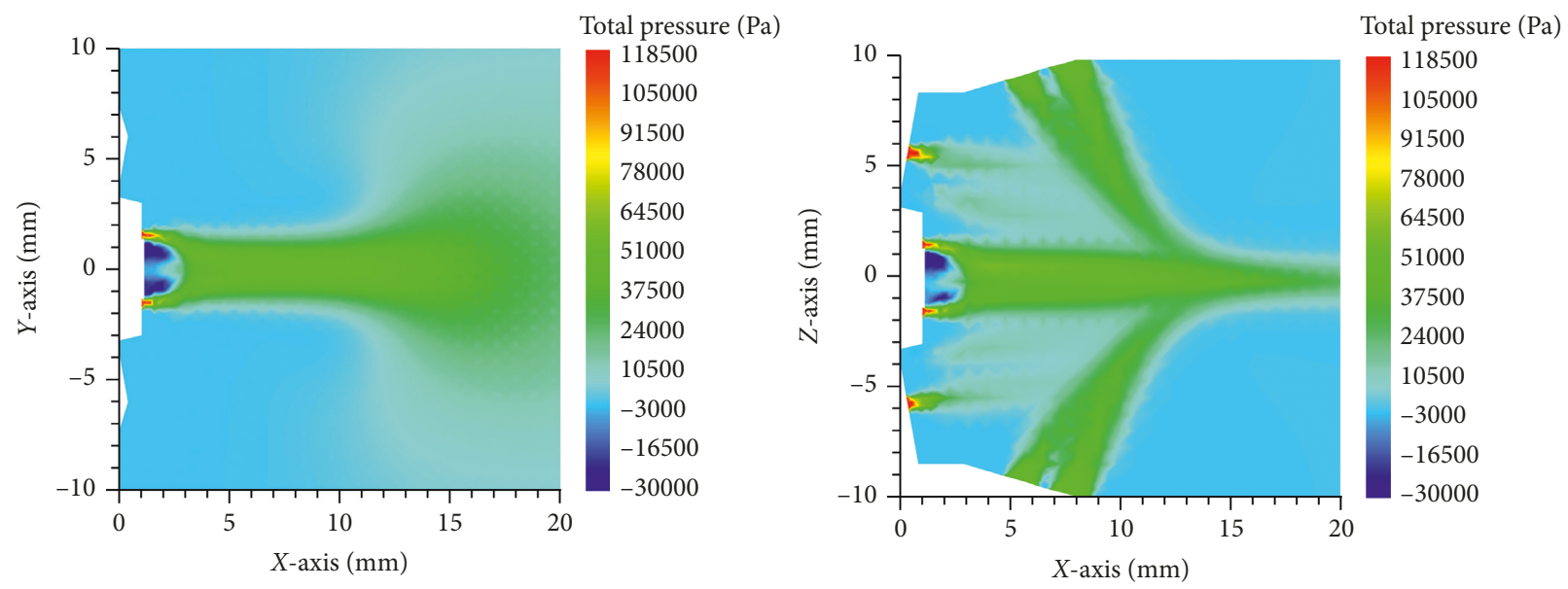

(b)

Figure 8: Comparison of spatial distribution of pressure field. (a) Pressure contours for single-hole nozzle: $X-Y$ plane (left); $X-Z$ plane (right). (b) Pressure contours for multiorifice nozzle: $X-Y$ plane (left); $X-Z$ plane (right).

small droplets. This problem can be improved by the introduction of auxiliary gas orifices.

\subsection{Gas-Liquid Two-Phase Flow Field}

4.2.1. Droplet Size Distribution. Figure 9 indicates the histogram of experimental and calculated drop size distributions. Figure 9(a) shows the experimental size distribution of the atomized droplets with the size range mainly between $2.31 \mu \mathrm{m}$ and $74.82 \mu \mathrm{m}$. Figure 9(b) presents the calculated results based on the DPM model, in which the droplet size range is mostly between $26.6 \mu \mathrm{m}$ and $70.13 \mu \mathrm{m}$. Due to the droplet collision and coalescent effect, both the experiment and simulation have larger particles with a diameter larger than $150 \mu \mathrm{m}$. Although the calculated results match the experimental data well, the significant divergence also exists for the smaller particles. The finer droplets with size between $2.31 \mu \mathrm{m}$ and $26.6 \mu \mathrm{m}$ take certain percentage for experiments. This is because some droplets dissolved in the spray filed in the experimental setup. These phenomena can not be calculated by the present model. Generally, as indicated in Figure 9(c), particle size distributions of the experiment and simulation are consistent. Both of them have the Sauter mean diameter (defined as the ratio of volumesurface mean diameter) around $55 \mu \mathrm{m}$.

4.2.2. Droplet Spatial and Size Distributions. In Figure 10, the horizontal coordinate presents the location of the droplet particles, and the vertical coordinate presents the diameter of the particles. As we can see from Figure 10(a), according to the particle spatial distribution on the plate, the shape of the multiorifice spray is oval. From the $Y$-axis, the droplets are dispersed between -25 and $25 \mathrm{~mm}$. From the $Z$-axis, the droplets are concentrated between -10 and $10 \mathrm{~mm}$. In order to compare, a further simulation analysis for the spray with single orifice is conducted. In Figure 10(b), without the auxiliary gas orifices and fan gas orifices, the atomization effect deteriorates, the larger droplet increases, and most of the droplets are concentrated in a circular region with radius about $10 \mathrm{~mm}$.

\section{Conclusions}

A new nozzle structure which can improve the spray coating uniformity was proposed in the present paper. Both the 


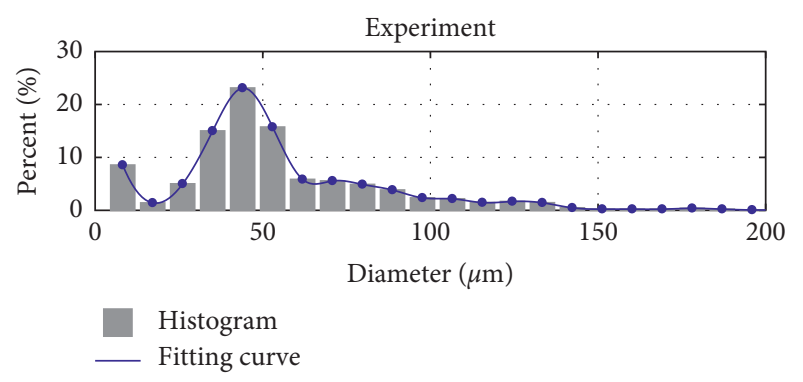

(a)

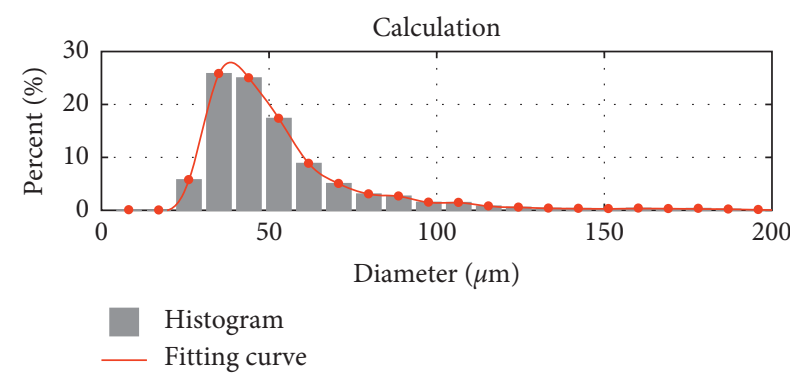

(b)

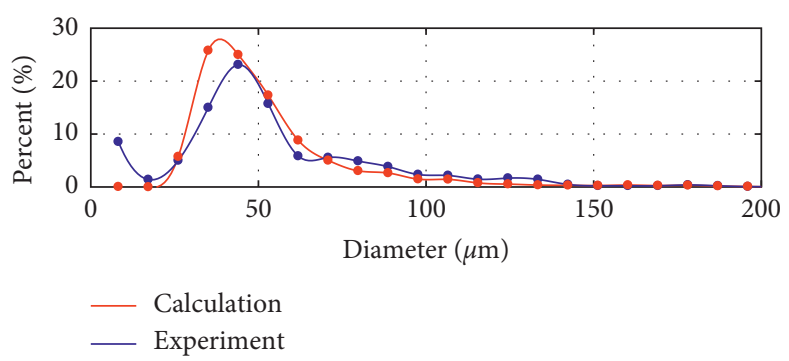

(c)

Figure 9: Droplet size distribution. (a) Histogram of experimental drop size distribution, (b) histogram of calculated drop size distribution, and (c) comparison of experimental data with calculated results.
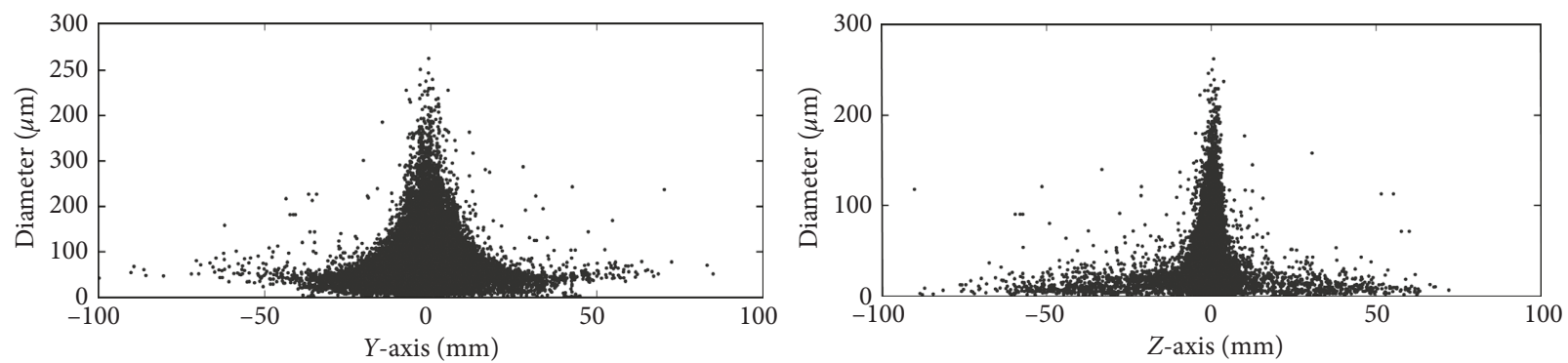

(a)
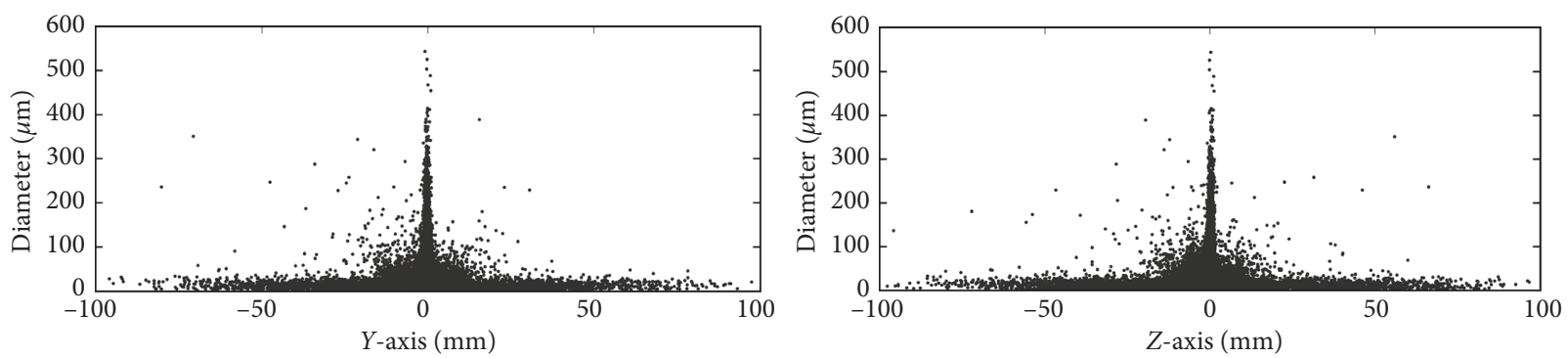

(b)

Figure 10: Droplet size and spatial distributions. (a) Droplet spatial and size distributions for multiorifice nozzle: $Y$-axis (left); $Z$-axis (right). (b) Droplet spatial and size distributions for single-hole nozzle: $Y$-axis (left); $Z$-axis (right).

experimental measurements and simulation were introduced to present the effects and characteristics of the new nozzle. The spatial distribution of pressure and velocity field and the droplet spatial and size distributions have been discussed in detail. With the auxiliary gas orifices and fan gas orifices, the multiorifice nozzle can provide the advantages of even distribution of droplets, alleviation of the overspray phenomenon in the central spray, and improvement of the spray efficiency with wider spatial distribution.

\section{Data Availability}

The data used to support the findings of this study are available from the corresponding author upon request. 


\section{Conflicts of Interest}

The authors declare that there are no conflicts of interest regarding the publication of this paper.

\section{Acknowledgments}

This work was supported by the National Natural Science Foundation (Grant nos. 11632016 and 11372006), Zhejiang Provincial Natural Science Foundation (LY17A020008), and Young Talent Cultivation Project of Zhejiang Association for Science and Technology (2016YCGC013).

\section{References}

[1] M. Bastwros and G. YongKim, "Ultrasonic spray deposition of $\mathrm{SiC}$ nanoparticles for laminate metal composite fabrication," Powder Technology, vol. 288, pp. 279-285, 2016.

[2] S. Melzig, D. Niedbalka, C. Schilde, and A. Kwade, "Spray drying of amorphous ibuprofen nanoparticles for the production of granules with enhanced drug release," Colloids and Surfaces A: Physicochemical and Engineering Aspects, vol. 536, pp. 133-141, 2018.

[3] K. Okuyama and I. W. Lenggoro, "Preparation of nanoparticles via spray route," Chemical Engineering Science, vol. 58, no. 3-6, pp. 537-547, 2003.

[4] L. J. Qian, J. Z. Lin, and H. B. Xiong, "Modeling of nonNewtonian suspension plasma spraying in an inductively coupled plasma torch," International Journal of Thermal Sciences, vol. 50, no. 8, pp. 1417-1427, 2011.

[5] N. P. Padture, M. Gell, and E. H. Jordan, "Thermal barrier coatings for gas-turbine engine applications," Science, vol. 296, no. 5566, pp. 280-284, 2002.

[6] L. J. Qian, J. Z. Lin, and M. Z. Yu, "Parametric study on suspension behavior in an inductively coupled plasma," Journal of Thermal Spray Technology, vol. 22, no. 6, pp. 1024-1034, 2013.

[7] S. Lee and S. Park, "Spray atomization characteristic of a GDI injector equipped with a group hole nozzle," Fuel, vol. 137, pp. 50-59, 2014.

[8] J. C. Lasheras, E. Villermaux, and E. J. Hopfinger, "Break-up and atomization of a round water jet by a high-speed annular air jet," Journal of Fluid Mechanics, vol. 357, pp. 351-379, 1998.

[9] L. J. Qian, J. Z. Lin, H. B. Xiong, and T. L. Chan, "Effects of operating conditions on droplet deposition onto surface of atomization impinging spray," Surface and Coatings Technology, vol. 203, no. 12, pp. 1733-1740, 2009.

[10] L. J. Qian and X. Y. Chu, "Particle properties for suspension plasma spray," International Journal of Numerical Methods for Heat \& Fluid Flow, vol. 24, no. 6, pp. 1378-1388, 2014.

[11] A. Aliseda, E. J. Hopfinger, J. C. Lasheras, D. M. Kremer, A. Berchielli, and E. K. Connolly, "Atomization of viscous and non-Newtonian liquids by a coaxial, high-speed gas jet: Experiments and droplet size modeling," International Journal of Multiphase Flow, vol. 34, no. 2, pp. 161-175, 2008.

[12] L. J. Qian, J. Z. Lin, and F. B. Bao, "Numerical models for viscoelastic liquid atomization spray," Energies, vol. 9, no. 12, p. 1079, 2016.

[13] C. L. Rivera, "Secondary breakup of inelastic non-Newtonian liquid drops," Ph.D. thesis, Purdue University, West Lafayette, IN, USA, 2010.

[14] H. B. Xiong, J. Z. Lin, and Z. F. Zhu, "Three-dimensional simulation of effervescent atomization spray," Atomization and Sprays, vol. 19, no. 1, pp. 75-90, 2009.
[15] N. Ashgriz, Handbook of Atomization and Sprays: Theory and Applications, Springer, New York, NY, USA, 2011.

[16] P. K. Senecal, D. P. Schmidt, I. Nouar, C. J. Rutland, R. D. Reitz, and M. L. Corradini, "Modeling high-speed viscous liquid sheet atomization," International Journal of Multiphase Flow, vol. 25, no. 6-7, pp. 1073-1097, 1999. 


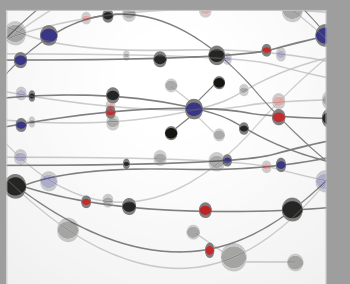

The Scientific World Journal
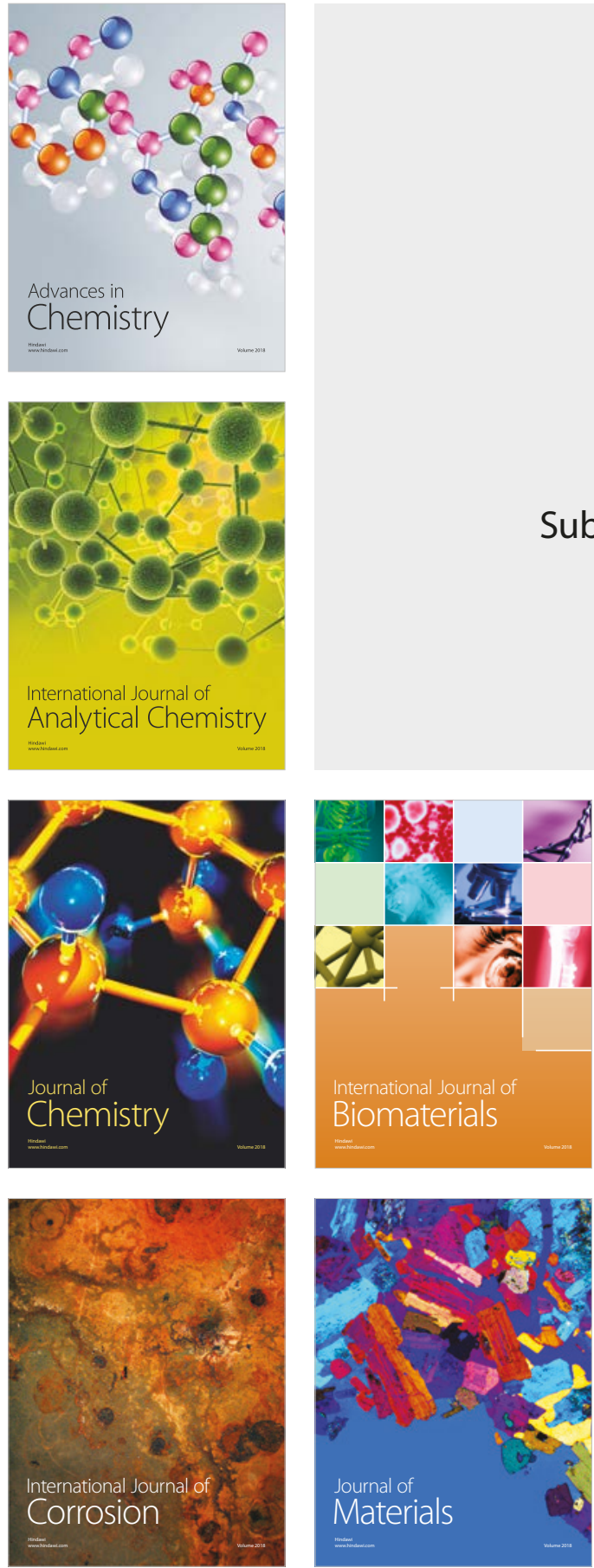

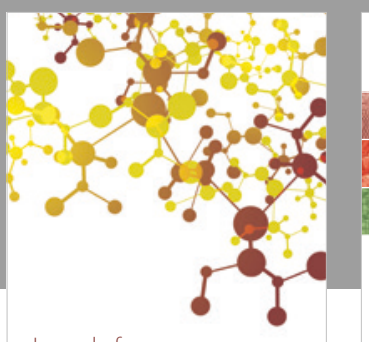

Journal of

Applied Chemistry
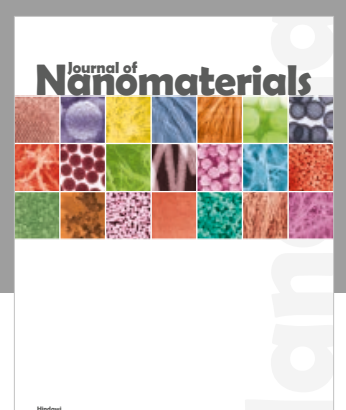

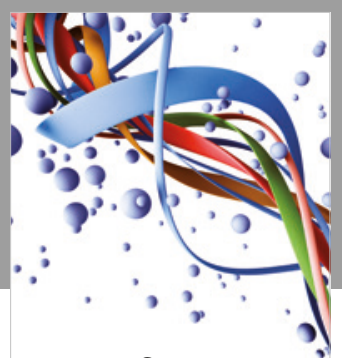

Scientifica

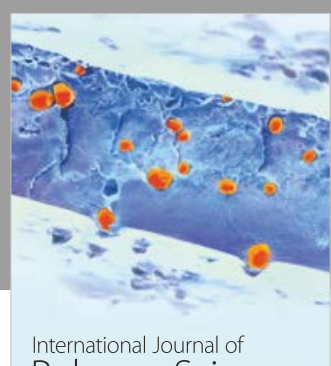

Polymer Science

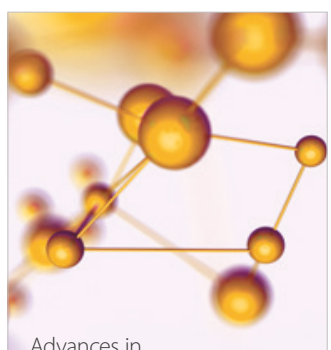

Physical Chemistry
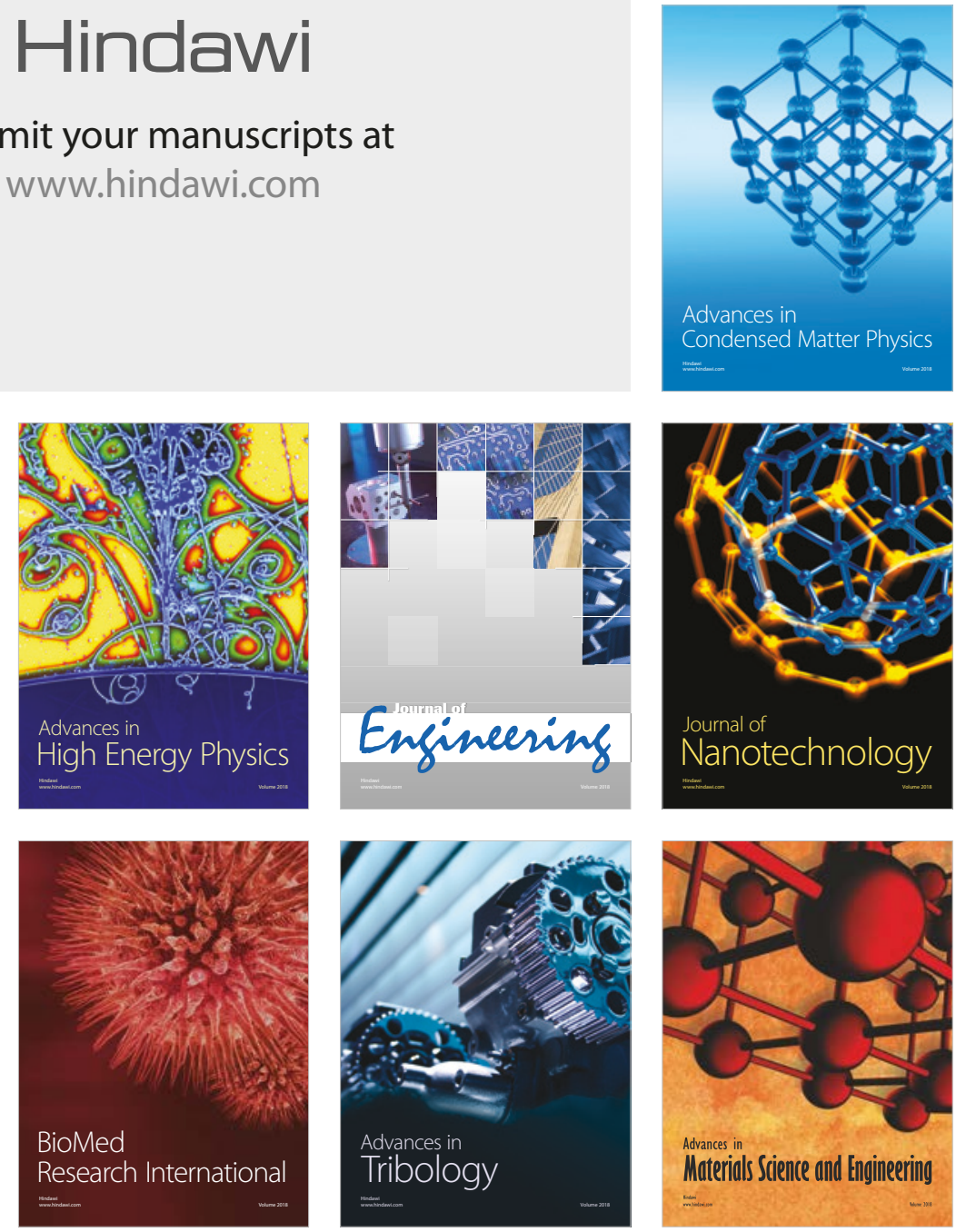